\title{
REALIZATION OF TEST PAPER SCORE CUMULATING SYSTEM BASED ON DIGITAL IMAGE PROCESSING
}

\author{
Simran Narang \\ Department of CSE \\ AIET, Jaipur, Rajasthan, India
}

\begin{abstract}
In order to solve the problem of handwritten score cumulating in test papers, this paper studies the implementation of test paper score cumulating system based on digital image processing on MATLAB 7.0.8 (R2009a) programming platform. The whole experiment process includes image preprocessing, scoring place positioning and segmentation, character recognition based on artificial neural network and the summation of scores. The experimental results prove that the method is effective and accurate, and also shows that MATLAB is effective in the application of this method.
\end{abstract}

Keywords: test paper score cumulating system, digital image processing, artificial neural network, Matlab.

\section{INTRODUCTION}

In the national education industry, increasingly more software applications and practical systems are applied to daily work in order to alleviate the burden of teaching and administrative staff, and improve their working efficiency. In the current marking model, despite the existence of card reading machines, subjective questions cannot be completely abandoned, the task of teachers is still arduous. They have to make judgements whether answers are correct or reasonable, and add up the scores of each coordinate system. The plane includes two important parameters, one of which is hue, and the other is saturation. Hue rotates around the vertical coordinate). Six standard colors are marked in Figure 1.1, with adjacent pure colors separated by 60 degrees. Saturation changes along the horizontal direction, with the lowest saturation at the origin of the coordinate system. Valueis located on the vertical axis of the coordinate system, changing continually along the vertical direction[3] question. There is no doubt that it will take up a lot of time in score accumulation. As a result of reducing the teachers' working efficiency virtually, the system proposed in this paper can effectively solve the problem of handwritten score summarization. Compared with the manual

\author{
Sapna Singh \\ Department of CSE \\ AIET, Jaipur, Rajasthan, India
}

summary of the scores, the test paper scores accumulation system achieves higher accuracy and reliability with high speed.

\section{A. Basic principle of Houghtransform}

Hough Transform is a voting principle of the parameter estimation technology, mainly used to extract geometric shapes with uniform characteristics from an original image. It projects all the points on a straight line or a curve of the original image to the intersection of all the lines in a parameter space. In the parameter space, the points are simply accumulated in the segmented accumulator units, and the peaks of the accumulator units are searched. The positions in the original image corresponding to the accumulator units in the parameter space where these peaks locate are the presence of straight lines or curves, whereby straight lines or curves in the image can be detected [1]. In fact, the essence of Hough transform is to cluster the pixels with a certain relation in the original image space, and find the corresponding parameter space accumulation points [2] which can associate the pixels with an analytical form.

\section{B. Searching red characters based on HSVmodel}

The HSV color model is evolved from the CIE 3D color space. In the HSV color model, each color is represented by Hue, Saturation, and Value. As shown in Fig.1, the hexagonal pyramid is the three-dimensional representation of this color model. In this model, the bottom surface of the hexagonal pyramid is located on the horizontal plane of coordinate system. The plane includes two important parameters, one of which is hue, and the other is saturation. Hue rotates around the vertical coordinate). Six standard colors are marked in Figure 1.1, with adjacent pure colors separated by 60 degrees. Saturation changes along the horizontal direction, with the lowest saturation at the origin of the coordinate system. Valueis located on the vertical axis of the coordinate system, changing continually along the vertical direction[3]. 


\section{International Journal of Engineering Applied Sciences and Technology, 2020 \\ Vol. 4, Issue 10, ISSN No. 2455-2143, Pages 236-241 \\ Published Online February 2020 in IJEAST (http://www.ijeast.com)}

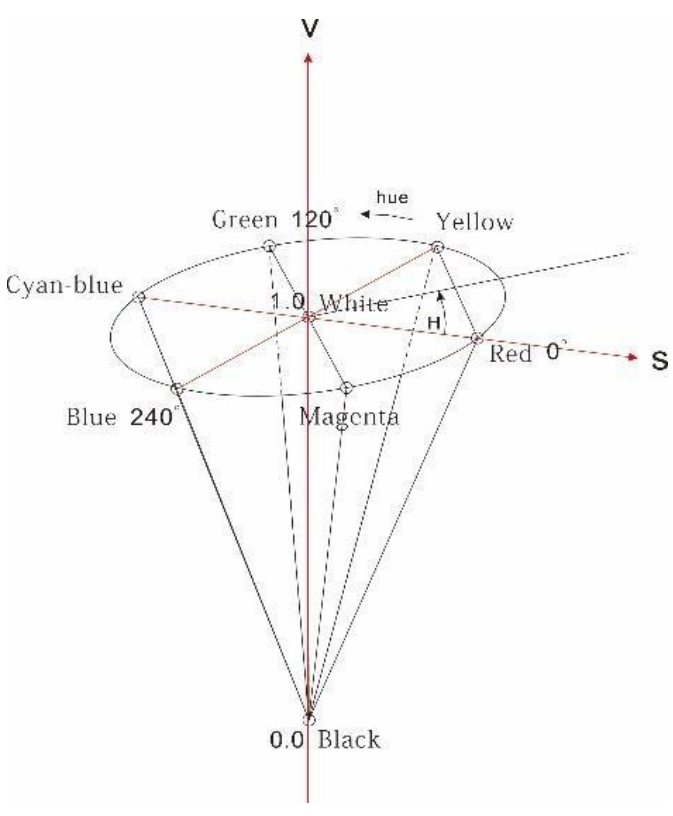

Figure. 1. Three-dimensional HSV hexagonal pyramid.

The following nonlinear transformation can change the RGB color space into HSV color space [4]. It may arise singular points and unstable fixed points.

\section{Introduction toANN}

ANN is a mathematical model that imitates the structure and thinking ways of brains. ANN can be used to solve some difficult problems. It is a hotspot branch of the artificial intelligence field. ANN algorithms learn and analyze through data and functions, apply appropriate treatments to become smarter. ANN must have neurons, and these neurons are connected to each other to form a neural network structure. ANN's learning and training process need to be remembered. Such memory is represented through the value of synapses which exist among neurons. Compared with other computational models, ANN has a great advantage in information distributed storage and parallel processing, which greatly improves the efficiency of information processing. Actually, ANN is a nonlinear dynamic system; the connecting structures of networks have impact on the learning and training abilities in varying degrees. View from the relationship between the various variables in the system, the network memory and recognition modes are increase functions of the number of neurons [6]. The performance of ANN is affected by four main factors, namely, the number of neurons, connections, network topology and learning methods.

\section{BPalgorithm}

BP neural network is essentially an error back propagation algorithm of learning process. The learning process consists of two parts. One is the forward propagation of information, and the other is the error backward propagation process. For the forward propagation of information, the neurons in the input layer in the network model receive the input information first, and then transmit this information to the neurons in the hidden layer, at last transfer it to the output layer. Due to the influence of the neural network performance, the final output may be different from the expected result. At the same time, the error back propagation process begins, which is opposite to the direction of the forward training. The error of the result propagates from the output layer to the input layer, and modifies the connection synaptic values between the neurons in each layer. The whole process follows the error gradient descends principle. The two opposite directions of the propagation process execute alternately, in order to ensure that errors continue to decrease through the adjustment of the synaptic values .The error decreases until it reaches an acceptable value, and the training can be stopped. There are varieties of BP neural network models, and the most commonly used are node input and output model, role function model, error calculation model and self -learning model, etc.

\section{SCORE CUMULATING SYSTEM}

The specific step $\mathrm{s}$ of implementing the score cumulating system are as follows:

- Image input: Inputing the images to be processed into computer and the loading the images in the cumulating system.

- Image preprocessing: Including image conversion, denoising, image edge detection, line extraction and image rotatation, etc

- Region localization and segmentation: Positioning the approximate location of the handwritten scores and then dividing the rectangle region.

- Character segmentation: Separating each question's scores from the rectangle region, and then separating the numbers into several single characters.

- Single-character recognition: This step is based on the results obtained in the previous steps, analyzing each character image segmented in 


\section{International Journal of Engineering Applied Sciences and Technology, 2020 \\ Vol. 4, Issue 10, ISSN No. 2455-2143, Pages 236-241 \\ Published Online February 2020 in IJEAST (http://www.ijeast.com)}

previous step, extracting the features, recognizing numbers through the established neural network and finally determining the character category.

- Score summary: Calculate the scores of each question, summing all the scores and output the results.

The flow chart of the overall steps is shown in Figure 5 .

\section{A. Image preprocessing}

Image preprocessing is the first step of this experiment, which plays a vital role. The original images not only have the contour, edge and color in formation of the image, such information will also occupy a certain amount of storage space, which leads to a lower system running speed. Therefore, under normal circumstances, most of the time we choose to convert the format of the image in order to optimize the system and solve the problem more quickly and efficiently. For example, original image are converted to grayscale or binary images. Image preprocessing is necessary before the image analysis, so that the information contained in the image can be simplified, only in this way can we understand the objecteharacteristics of images more clearly and directly. The edges of the iimage refer to the part where the local brightness changes in the image is relatively obvious. They can fully reflect the image texture and the shapes of the basic features. In order to facilitate the use of Hough transform, the first step is image edge detection.

\section{B. Specific steps in imagepreprocessing}

- Converting the original images into binary images.

- Using canny operator to detect the edges of the images, and remove small and isolated fake edge segments.

- Using Hough transform to extract lines.

- Adjusting the lines into horizontal direction.

\section{Score region positioning and segmentation}

The localization and segmentation of the test score region is one of the most important steps in the implementation of the cummulating system. With the realization of this step, we can lock the exact location of the score region and segment the handwritten scores accurately. The results obtained in this process are used in the subsequent steps, so the accuracy of the segmentation is directly related to the final recognition rate of the whole system.

\section{- Score are positioning}

In order to ensure successful test score positioning and segmentation, we observe the basic characteristics of the images. Teachers use red signature pens when marking test papers. The color property of the score areas is obviously different from that of the surrounding areas, so it is convenient to locate the score area. Firstly, the original image is converted from RGB to HSV, and the three components of $\mathrm{H}, \mathrm{S}$ and $\mathrm{V}$ are obtained. The three basic components of red color are obtained by referring to literatures. By limiting the three component at the same time, the location of score areas can be found. Due to the problems of image quality and clarity, a morphological noise filter consisting of two basic operations which are opening and closing, is applied here to remove small objects that are not related to the object.

\section{- Score region division}

Once a score area has been locked, the $\mathrm{X}$ and $\mathrm{Y}$ coordinate values of the score area are stored, and the minimum and maximum values in both direction are recorded, which can be used to cut apart the rectangle area within which the scores are located. The flow chart of score area positioning and segmentation is shown in Figure 2.

\section{D.Character Segmentation}

The segmentation of a single character plays a transitional role in the system. The basic idea of this step is to use the matrix projection method, in order to determine whether the $\mathrm{X}$ direction exists the score character pixels or not. Figure 2. shows the projection of a matrix of a score region, with each element in the matrix being either 0 or 1 . The projection of this matrix is a one-dimensional vector, which deposits both nonzero elements, and 0 elements. The location of the 0 elements means that the corresponding position has no character pixels. Therefore, characters can be split based on 0 and non-0element positions. For the images in the experiment, the scores of each question are different, with both single-digit scores and two- digit scores. Therefore, we not only need to separate the scores of all questions, but also to separate the ten digit numbers of each question's score. In the experiment, the scores of questions should be separated first, and then the digital numbers should be segmented. In order to distinguish the segmentation of the question score and segmentation of a single number, a 1-dimensional array is defined to record the distance between all the characters presented in the image, and then averages of these values stored in the array. We consider the 


\section{International Journal of Engineering Applied Sciences and Technology, 2020 \\ Vol. 4, Issue 10, ISSN No. 2455-2143, Pages 236-241 \\ Published Online February 2020 in IJEAST (http://www.ijeast.com)}

characters to be in different questions if the distance of adjacent characters is longer than the mean distance, and as for distance values smaller than or equal to the mean, we consider the adjacent characters come from the same question's score. The decision is expressed as follows

where

- $\quad D_{i}$ represents the $\mathrm{i}$-th interval in the projected matrix;

- $\quad n$ is the number of total intervals.

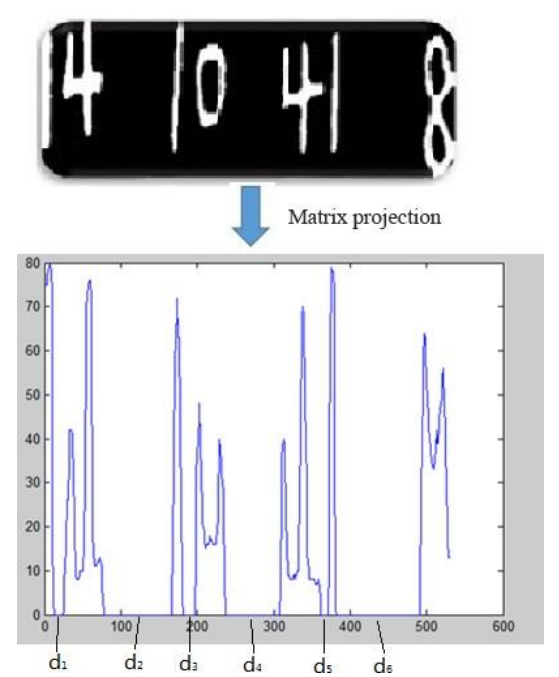

Figure.3. Matrix projection results.

\section{E. CharacterRecognition}

In the implementation of the overall system, character recognition is a very important part. It determines whether the final result is correct or not. There are many kinds of methods that we can use. The most common method is the template matching algorithm and the ANN algorithm. By comprehensively analyzing the accumulation system and considering that the test scores are handwritten, we conclude that the success rate of the template matching algorithm will not be high. In order to ensure the accuracy of the character recognition and the final results, a self-coding neural network is applied in this paper. whichis based on BP neural network algorithm. The selfcoding neural network algorithm in the experiment uses a single-character image and its Gabor feature as the input node of the network for single character recognition. We construct each individual character image obtained in the previous steps. A self-coding neural network is used to learn each image. By using the weights of the established network, the image features of each character in the image are reconstructed. Finally, the correlation between the sample and the true final result are calculated. The maximum correlation value is the result of character recognizing.

\section{F. ScoreSummary}

Score summation is the final step of the experiment. The main task in this process is to calculate the true score of each question, and finally sum the scores and output the results. The input and output of another successful case are shown in Figure 4.
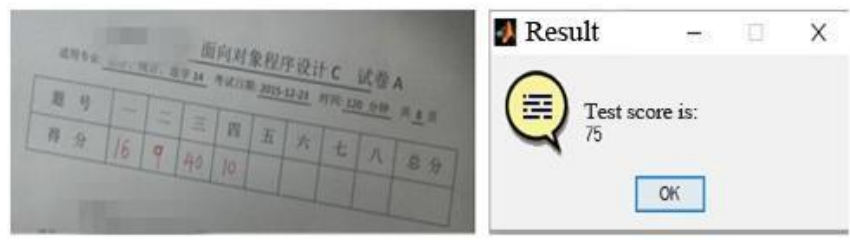

Figure.4. Another successful case

\section{II CONCLUSION}

The final realization process of accumulation system, the workload of teachers is reduced. Besides, the accuracy of the results of the cumulating system is high as the reasonable image processing methods have been used, including characters division, location normalization methods, and neural network. In the meantime the experimental program is relatively simple with highefficiency under certain requirements.

\section{REFERENCES}

[1].[Online].Available:http://wenku.baidu.com/view/e25dd f77 e009581b6bd9ebbd.html? From=search (In Chinese)

[2]. Zhou Xiaodong, Li Ya jie, Yue Xiaofeng, et al. (2010) Study on the Method of Automobile Instrument Pointer Recognition [J]. Journal of Changchun University of Technology, vol. 31, no. 1. pp. $32 \sim 36$ (In Chinese)

[3][Online]. Available:http://wenku.baidu.com/view/4 94b9 d29bd64783e09122bec.html (In Chinese)

[4][Online].Available:

http://wenku.baidu.com/view/3856f3adaef8941ea76e

05a1.html (In Chinese)

[5]. Grossberg S. Nonlinear neural networks: principles, mechanisms, and architectures, Neural Network, 1

[6]. Chua L., Yang L. (1988) Cellular neural network: Theory and Applicalions. IEEE Transactions on Circuits Systems I, , vol. 35, no. 10. pp. 1273 - 1290 
[7]. Jing G, Du W, Guo Y. (2012) Studies on prediction of separation percent in electrodialysis process via BP neural separation and improved BP algorithm. Desalination, vol. 291, no. 2. pp. 78-93.

[8]. MAO Qing-hua. (2003) Application of Artificial Neural Network in Aerospace Experiment Effectiveness Evaluation. Automation of Theory \& Applications.

[9]. Yang, Cheng-Hsing. "Inverted pattern approach to improve image quality of information hiding by LSB substitution Pattern Recognition 41, no. 8 (2013): 2674-2683.
[10]. Sancheti, Ankita. "Pixel Value Differencing Image Steganography Using Secret Key." International Journal of Innovative Technology and Exploring Engineering (IJITEE) ISSN (2012): 2278-3075.

[11]. Amirtharajan, R., D. Adharsh, V. Vignesh, and R. John Bosco Balaguru. "PVD blend with pixel indicatorOPAP 